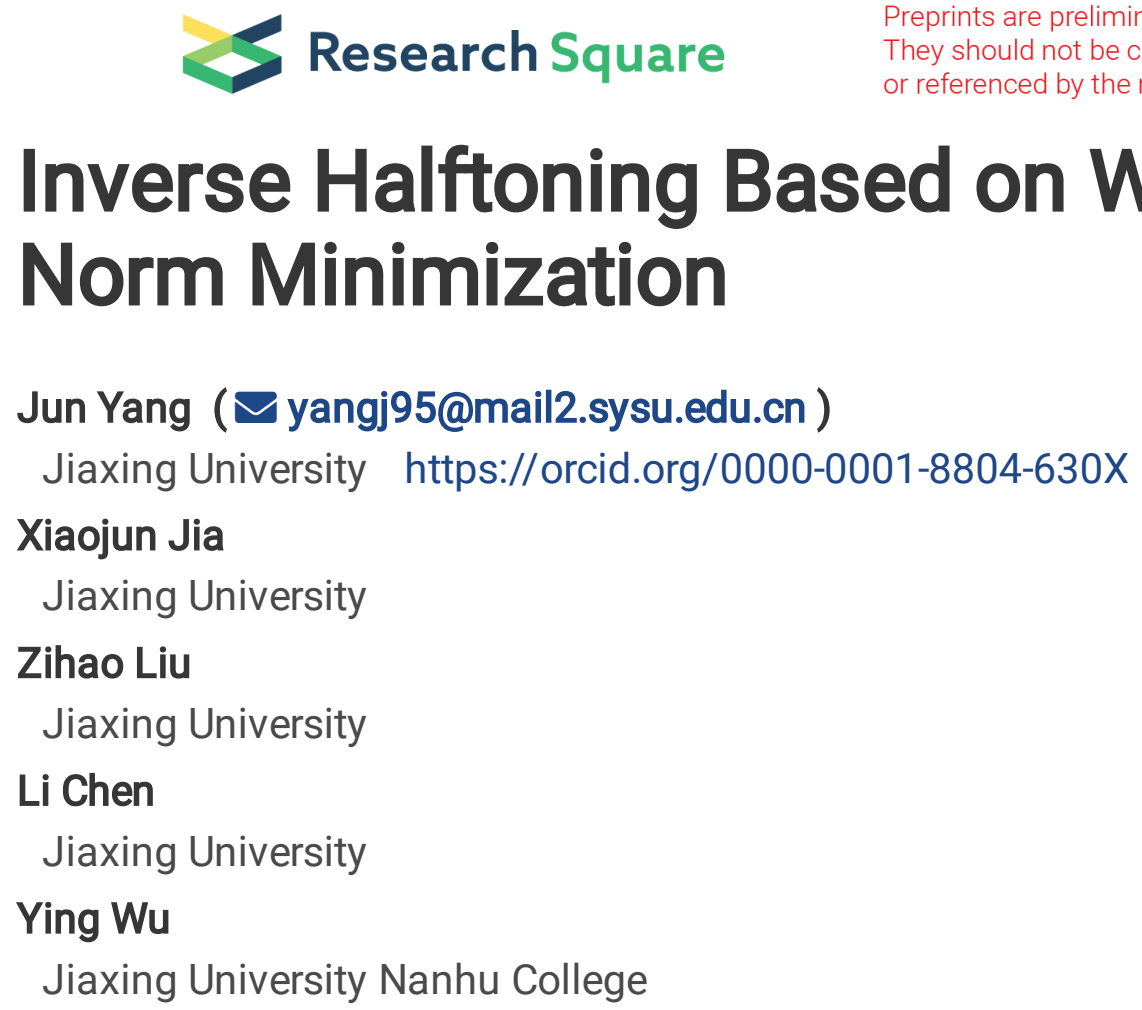
Norm Minimization

\title{
Inverse Halftoning Based on Weighted Nuclear
}

\section{Research Article}

Keywords: Inverse halftoning , Weighted nuclear norm minimization, Nonlocal similarity , Alternative projections

Posted Date: May 17th, 2021

DOl: https://doi.org/10.21203/rs.3.rs-507071/v1

License: (c) (i) This work is licensed under a Creative Commons Attribution 4.0 International License. Read Full License 


\title{
Inverse Halftoning Based on Weighted Nuclear Norm Minimization
}

\author{
Jun Yang · Xiaojun Jia • Zihao Liu • Li \\ Chen · Ying Wu
}

Received: date / Accepted: date

\begin{abstract}
The inverse halftoning technology refers to restore a continuoustone image from a halftone image with only bi-level pixes. However, recovering the continuous images from their halftoned ones is normally ill-posed, which making the inverse halftoning algorithm very challenging. In this paper, we propose an optimization model with two alternate projections (TAP) for image inverse halftoning under the weighted nuclear norm minimization (WNNM) framework. The main contributions are two-folds. First, the WNNM nonlocal regularization term is established, which offers a powerful mechanism of nonlocal self-similarity to ensure a more reliable estimation. Second, the alternate minimization projections are formulated for solving the image inverse halftoning, which reconstructs the continuous-tone image without destroying the image details and structures. The experiment results shown that the proposed method outperformed the state of the arts in terms of both objective measurements and subjective visual performance.
\end{abstract}

Keywords Inverse halftoning - Weighted nuclear norm minimization . Nonlocal similarity · Alternative projections

\section{Introduction}

The halftoning algorithm can represent a gray scale image with only one color through the use of ink dots, and is widely used in today's digital image printing, publishing and displaying applications, such as printers, newspapers, books, magazines, etc. $[11,13]$. Most printing devices are based on binary technologies. Halftoning involves use of patterns or ink dots at a high spatial

Jun Yang, Xiaojun Jia, Zihao Liu and Li Chen

College of Information Science and Engineering, Jiaxing University, Jiaxing, China

E-mail: yangj95@mail2.sysu.edu.cn, xjjiad@sina.com

Ying $\mathrm{Wu}$

College of Information Engineering, Jiaxing Nanhu University, Jiaxing, China 
frequency to simulate to the eye an integrated gray scale image. Halftoning techniques are also used to render electronic color images on common electronic display devices that use color mapping to limit the number of bits per pixel and thereby reduce memory requirements. Halftone images are typically difficult to manipulate for the missing information of image. Some image processing procedures, including scaling, compression, and enhancement could cause image quality degradation severely [14]. In order to avoid the degradation, gray image is necessary to be reconstructed from the haltone version by using inverse halftoning technology.

It is difficult to process or display the halftone images directly. Therefore, as illustrated as in Fig. 1, the halftoned images are often inverse halftoned. Considering that the inverse halftoning procedure is an operation mapping $\{0,1\}^{H \times W}$ onto $\mathbb{R}^{H \times W}$, where $H$ and $W$ are the image height and width respectively. The inverse halftoning technique obviously belongs to the ill-posed inverse problems, which is rather challenging. In the past several decades, a lot of image inverse halftoning methods were proposed to utilize the local smoothness prior of images and have achieved good inverse halftoning results, which include tree-structured vector quantization method [24], convex set projection method [8], iterative statistical smoothing method [25]. However, these methods tend to produce fuzzy results while maintaining local smoothness, which leads to the contradiction between preserving edges and textures. A balance needs to be considered between preserving edges and textures. The filtering methods,such as nonlinear permutation filtering [12] and anisotropic filtering [15], often focus on computational complexity rather than image visual quality. Although the Bayesian approaches $[17,23]$, look-up-table (LUT) based approaches [7], shearlet representation and wavelet-based approaches $[2,18,20,26]$ boost the visual performance of recovered image, but details and structures of reconstruct images can not well reserved. Targeting at above problems, the recently proposed Block-matching and 3D filtering (BM3D) based method [16] established a optimization function with a regularization term of nonlocal similarity, and achieved the state-of-the-art performance. However, there are one main problem that restricts the performance. The BM3D-based method highly relied on a sophisticated deterministic annealing optimization [22], which needs to denoise recovered images iteratively. Hence, the output of the method usually consists of visible artifacts.

In order to resolve the issue above, we minimize the residual errors between the input halftoned image and the re-halftoned version of its reconstructed image in each iteration (the first projection) so as to maintain local smoothness. Followed the first projection we add a new constraint term expressed via the weighted nuclear norm minimization (WNNM) optimization (the second projection) to improve the self-similarity prior of nonlocal image patches. By gathering similar nonlocal image patches as a group and then limiting the singular values of each group via the sophisticated parameters, our TAP-WNNM constraint can construct a low-dimensional manifold space from each image group. As a result, there are distinct patterns in each group, such as the contours of an animal's face, that can be extracted, while the artifacts can also 


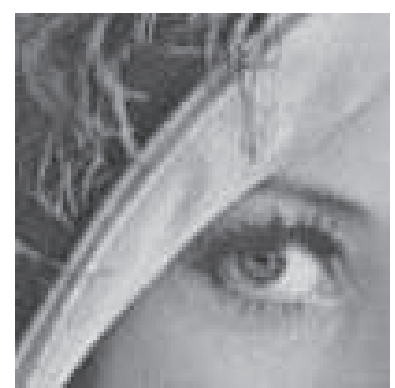

(a)

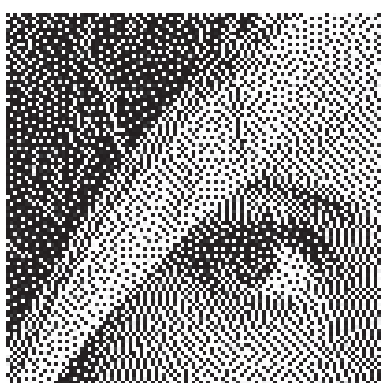

(b)

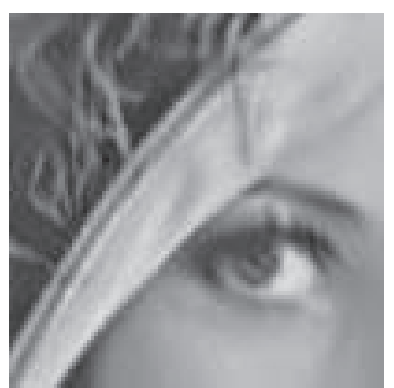

(c)

Fig. 1: Examples of halftone and inverse halftoning images. Detail of the Lena image: original (a), error diffusion halftoned (b) and inverse halftoned (PSNR $=33.14 \mathrm{~dB})(\mathrm{c})$.

be well separated. Therefore, the similarity of non-local patches in this group of images can be enhanced. In order to optimize the whole framework, we also use TAP-WNM to construct an iterative projection method to ensure the lower bound of each group of singular values. Experimental results show that this method has good performance.

The rest of this paper is organized as follows. In Section 2, we introduce the related work. Section 3 presents the proposed framework. In Section 4, experiment results are reported. Finally, conclusions are addressed in Section 5 .

\section{Related Work}

\subsection{Weighted Nuclear Norm Minimization Regulation}

The weighted nuclear norm minimization (WNNM) regulation $[5,6]$ is the generalization form to the nuclear norm minimization(NNM) model [1]. WNNM is described as:

$$
\min _{\mathbf{X}}\|\mathbf{Y}-\mathbf{X}\|_{F}^{2}+\|\mathbf{X}\|_{\mathbf{w}, *}
$$

where $\|\mathbf{X}\|_{w, *}=\sum_{i} w_{i} \sigma_{i}(\mathbf{X})$ is the weighted nuclear norm (WNN) of ma$\operatorname{trix} \mathbf{X}$, and $\mathbf{w}=\left[w_{1}, \cdots, w_{n}\right]^{\mathrm{T}}\left(w_{i} \geq 0\right)$ is the weight vector. The $\sigma_{i}(\mathbf{X})$ is the $i$ th singular value of $\mathbf{X}$. The Corollary 1 in [6] proved that Eq. (1) would have a closed-form solution if the weights are monotonic increasing:

$$
\hat{\mathbf{X}}=\mathbf{U} S_{w / 2}(\boldsymbol{\Sigma}) \mathbf{V}^{\mathrm{T}},
$$

where $\hat{\mathbf{X}}=\mathbf{U} \boldsymbol{\Sigma} \mathbf{V}^{\mathrm{T}}$ is the singular value decomposition (SVD) [3] of $\hat{\mathbf{X}}$ and $S_{w / 2}(\bullet)$ is the soft-thresholding operator typically generated by the weight vector $\mathbf{w}$ :

$$
S_{w / 2}\left(\boldsymbol{\Sigma}_{i i}\right)=\max \left(\boldsymbol{\Sigma}_{i i}-w_{i} / 2,0\right) .
$$


The WNNM model has demonstrated the competitive performance for image denoising. However, if we directly extend WNNM to image inverse halftoning by denoising the inversed halftoning version, inverse halftoning and denoising artifacts may happen, which will result in the details and textures missing. In this paper, we propose an optimization model with two alternate projections (TAP) for image inverse halftoning under the WNNM framework, which preserves the construction and texture details of the inverse halftoning image while removing the artifacts and halftoning noise.

\subsection{Image Inverse Halftoning}

During the last decade, a lot of approaches have been proposed for image inverse halftoning. Among them, Bayesian based approach [17] employs the least-mean-square (LMS) algorithm to build the map between the current processing position and its corresponding neighboring positions in each type of halftone image. The look-up-table (LUT) halftoning method [7] is an effective method for constructing halftone image and can approximate the dot distribution of the learned halftone image set. In the wavelet-based method [20], the convolution operator specified by the model is first half-modulated and then the residual noise is attenuated by scalar wavelet domain shrinkage. However, these methods only explore the internal information of halftone images to improve the performance of inverse halftone images.

There also exist some methods exploiting internal similarities of an image $[19,27]$. Sparsity-based inverse halftoning method [27] is proposed to solve the problem of cross-style image restoration from halftone image to continuous tone image. These works only using the internal information to inverse halftoning, which are generally inferior to external ones. Inspired by recen$\mathrm{t}$ success of deep learning technology in image processing applications, some convolutional neural network $(\mathrm{CNN})$ based works $[9,10]$ are developed, which explore the external information for halftone image. Using external halftone and continuous tone images, the CNN based inverse haltoning approach [9] trains a deep CNN as a nonlinear transformation function to map a halftone image to a continuous tone image. However, the external methods built their models directly from the low-quality inputs, without developing the internal similarities. Hence it remains a challenge to combine the internal and external priors for image inverse halftoning.

The BM3D based method [16] is a representative one, which added a regularization term of nonlocal similarity and significantly improve the performance. To avoid blurry solutions, The G-SVD based approach [28] is proposed to exploit the similarity of nonlocal image patches, which achieve the state-of-the-art performance.

This paper proposes an optimization framework with two alternate projections (TAP) for image inverse halftoning under the WNNM framework, which utilizes the strong low-rank prior of image non-local similar patches as the internal information. And the external information between the images of the 
adjacent iterations are also explored to enhance the performance of the inverse halftoning.

\section{The Inverse Halftoning Framework}

The proposed inverse halftoning model reconstructs the continuous image from halftone version while tries to preserve local smoothness under the constraint of nonlocal similarity. In this section we first explain how to utilize the unconstrained local smoothness (the first projection), and then discuss the proposed WNNM constraint (the second projection). Finally an iterative projection strategy is established upon TAP-WNNM to optimize the framework.

\subsection{Preserving Local Smoothness}

The halftoned image $\mathbf{Y}$ is generated from its continuous-toned version $\mathbf{X}$ by $\mathbf{Y}=\mathcal{H}(\mathbf{X})$ where $\mathcal{H}$ represents a generic halftoning model, such as dotdiffusion dithering or error diffusion. In this paper, the halftoning model is error diffusion. In order to preserve local smoothness, we look for an approximation $\hat{\mathbf{X}}$ which can optimally represent $\mathbf{X}$ :

$$
\min _{\hat{\mathbf{X}}}\|\mathbf{Y}-\mathcal{H}(\hat{\mathbf{X}})\|_{2}^{2}
$$

\subsection{Constraining Nonlocal Similarity With WNNM}

Obviously, the optimal result of Eq. (4) is an approximation $\hat{\mathbf{X}}$ which is exactly the same as the real continuous-tone image $\mathbf{X}$. However, it is usually impossible for that the optimization is non-convex and highly ill-posed. What's worse, a lot of works have proved that Eq. (4) often tends to preserve local-space-region smoothness of image and gets stuck in blurry solutions [16]. Therefore, in order to limit the solution set, avoid fuzzy results and better restore discontinuity, we add WNNM constraints to promote the non-local patch similarity:

$$
\min _{\hat{\mathbf{X}}}\|\mathbf{Y}-\mathcal{H}(\hat{\mathbf{X}})\|_{2}^{2}+\|\mathbf{X}\|_{\mathbf{w}, *}
$$

where $\mathcal{H}$ is the halftoning operation, and $\|\mathbf{X}\|_{w, *}=\sum_{i} w_{i} \sigma_{i}(\mathbf{X})$ is the weighted nuclear norm of matrix $\mathbf{X}, \mathbf{w}=\left[w_{1}, \cdots, w_{n}\right]^{\mathrm{T}}\left(w_{i} \geq 0\right)$ is the weight vector, and $\sigma_{i}(\mathbf{X})$ is the $i$ th singular value of $\mathbf{X}$. More specifically, $\|\mathbf{X}\|_{w, *}$ consists of a grouping phase, a WNNM phase and a pixel-averaging phase. For a reconstructed image $\hat{\mathbf{X}}$, firstly, similar image patches are grouped as $\|\mathbf{X}\|_{w, *}$, and singular values of each group are decomposed to synthesize weighted non-zero singular values. Finally, the average pixel value of overlapping image patches is calculated. Usually the components corresponding to the previous singular values represent the common pattern, while other components may be destroyed 


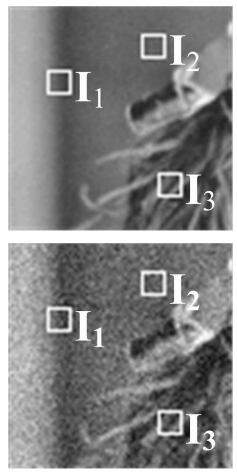

(a)

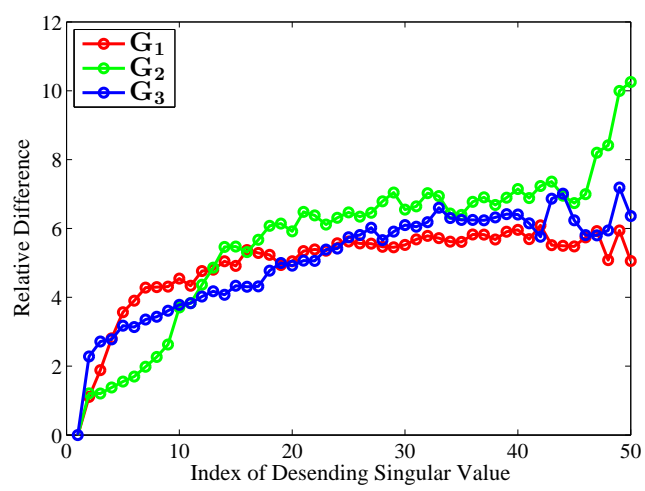

(b)

Fig. 2: The influence of noise on group singular values. (a) Top: the original image (Ori); Bottom: the image corrupted by Gaussian noises with mean 0 and standard deviation 15 (Corr). (b) (SV@Corr - SV@Ori)/SV@Ori, where SV@Ori and SV@Corr are singular values of groups in the two images respectively. The groups $\mathbf{G}_{\mathbf{1}}, \mathbf{G}_{\mathbf{2}}, \mathbf{G}_{\mathbf{3}}$ correspond to the reference patches $\mathbf{I}_{\mathbf{1}}, \mathbf{I}_{\mathbf{2}}, \mathbf{I}_{\mathbf{3}}$ individually.

by noise. The importance of significance patterns to halftone noise can be increased by constraining the leading singular values with large weights and other singular values with small weights, thus reducing artifacts and enhancing patch similarity.

We will discuss these three stages in detail below.

\subsubsection{Grouping Phase}

Intuitively, visually similar patches are usually concentrated near a manifold, while visually different patches are distributed differently. The purpose of the grouping stage is to separate spatially distant manifolds so as to find a good linear representation of each manifolds in the following decomposition stage. Similar ideas can be found in work related to denoising, for example [21].

For the given image with size of $H \times W$, every $h \times w$ adjacent pixels are extracted, aim to produce $(H-h+1) \times(W-w+1)$ normal patches of $h w$ dimensions. Among them $N$ reference patches covering the whole image are selected uniformly. For each reference patch $\mathbf{I}_{\mathbf{i}}$, finding the $C$ most similar regular patches in $p \times q$ surrounding pixels and sort them by Euclidean distance. These $C$ patches are further rearranged as an $h w \times C$ matrix with each column storing one of them sequentially.

In the conclusion, the grouping stage (GROUP) takes an image and the number of groups (i.e., $N)$ as the input and outputs an $h w \times C$-dimensional $N$ matrix. 


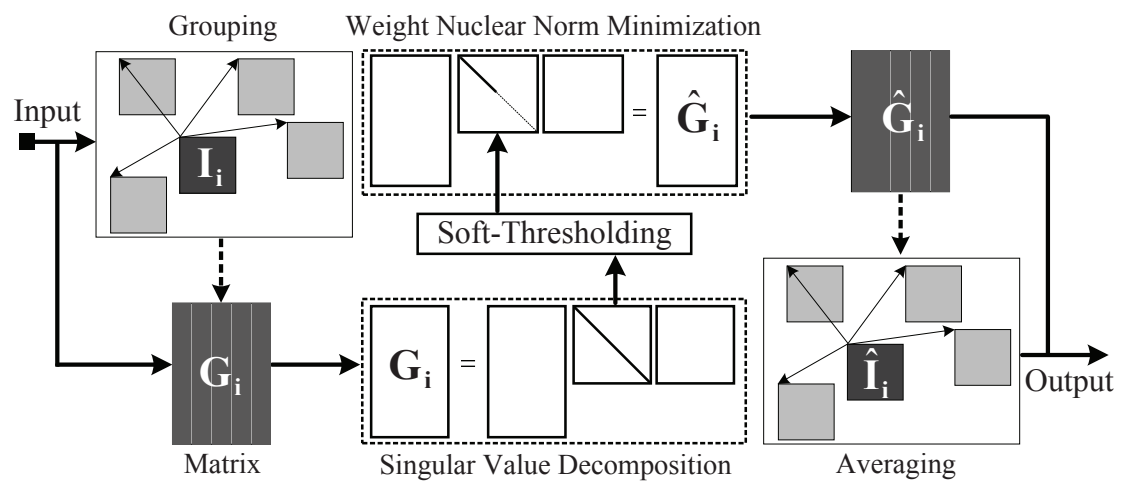

Fig. 3: The pipeline of a projection. $\hat{\mathbf{I}}_{\mathbf{i}}$ and $\hat{\mathbf{G}}_{\mathbf{i}}$ are the updated versions of $\mathbf{I}_{\mathbf{i}}$ and $\mathbf{G}_{\mathbf{i}}$ respectively.

\subsubsection{Weighted Nuclear Norm Minimization Phase}

Let $\left\{\mathbf{G}_{\mathbf{1}}, \mathbf{G}_{\mathbf{2}}, \ldots, \mathbf{G}_{\mathbf{N}}\right\}$ be an outcome of GROUP. We apply Weighted Nuclear Norm Minimization (WNNM) on each of the matrix and record the minimal weighted nonzero singular value: $d\left(\mathbf{G}_{\mathbf{i}}\right)=\min \left\{S_{w / 2}\left(\sigma_{1}\right), S_{w / 2}\left(\sigma_{2}\right), \ldots\right\}$, where $\sigma_{j}$ is a nonzero singular value of $\mathbf{G}_{\mathbf{i}}$. The output of the WNNM phase is defined as the minimum of $\left\{d\left(\mathbf{G}_{\mathbf{1}}\right), d\left(\mathbf{G}_{\mathbf{2}}\right), \ldots, d\left(\mathbf{G}_{\mathbf{N}}\right)\right\}$.

The goal of this phase is to describe the low-dimensional manifolds in each set in order to separate the significant patterns concentrated near the manifolds from the noise that is often orthogonal to the manifolds. Fig. 2 shows an illustration. After the image is destroyed by zero-mean Gaussian noise, we can see that the main singular values usually do not change much, while the rest of the singular values change a lot. Therefore, by limiting the lower bound of the nonzero minimum singular value to a large, the noise can be successfully ignored.

\subsubsection{Pixel-Averaging Phase}

For the overlapping patches in the patch grouping stage extracted above, each pixel may have several approximations with random halftone noise. It is natural to use their average as the new approximate pixel at this position. In this way, the main message is likely to be enhanced and false or rough textures are nicely removed.

The patches recovered in each $\mathrm{X}$ is put back to rebuild the input image with an average overlap of pixels (denote this process as "return"). We describe this phase in detail below.

The restored grouping patches $\hat{\mathbf{G}}_{\mathrm{p}}$ are the result of the patch grouping phase. The patch recovered at each $\hat{\mathbf{G}}_{\mathbf{p}}$ are put back, and the input image is reconstructed with average overlapping pixels (denote this procedure as "Back"). We will describe this phase in detail below. 
We define the following formula:

$$
\hat{\mathbf{G}}=\mathbf{R}(\hat{\mathbf{X}})
$$

where $\mathbf{R}(\cdot)$ is the extraction operator which extracts $\hat{\mathbf{G}}$ from image $\hat{\mathbf{X}}$ for patch $\mathbf{p}$, and the transpose of it is denoted by $\mathbf{R}^{\mathbf{T}}(\cdot)$. The vectorized patches can be put back to their corresponding positions in the reconstructed image as columns of $\hat{\mathbf{G}}$ and filled with zeros elsewhere.

Taking the average of all the matrices $\left\{\hat{\mathbf{G}}_{\mathbf{p}_{i}}\right\}, i=1,2, \cdots, N$, where $\mathbf{p}_{i}, i=$ $1,2, \cdots, N$ are the patches which are extracted from the image, the recovery of the whole image $\hat{\mathbf{X}}$ from $\left\{\hat{\mathbf{G}}_{\mathbf{p}_{i}}\right\}$ can be described as:

$$
\hat{\mathbf{X}}=\sum_{i=1}^{N} \mathbf{R}^{T}\left(\hat{\mathbf{G}}_{\mathbf{p}_{i}}\right) \oslash \sum_{i=1}^{N} \mathbf{R}^{T}\left(\mathbf{1}_{n \times m}\right)
$$

where $\oslash$ represents the element-wise division and $\mathbf{1}_{n \times m}$ is a matrix of size $n \times m$ with all elements 1 .

Fig. 3 shows the overall schematic diagram of the WNNM-constrained nonlocal similarity, including the grouping phase, WNNM and pixel-averaging phase. These three stages are in-place operation, such as extracting internal halftone image information.

\subsection{Iterative Projection}

Under unconstrained conditions, the residual errors in Eq. (5) can by optimized by iteratively perturbing $\hat{\mathbf{X}}$ around the decision boundary when $\mathcal{H}(\hat{\mathbf{X}})$ and $\mathbf{Y}[16]$ are inconsistent. We refer to this iteration as the coding step.

To handle the increased constraints, we insert an approximate update step after each coding step to ensure that the WNNM constraint is satisfied after projections.Given an approximate $\hat{\mathbf{X}}$, the projection is performed by the following steps:

1. Apply grouping on $N$ reference patches of $\hat{\mathbf{X}}$ to generate a set of matrices $\left\{\mathbf{G}_{1}, \mathbf{G}_{2}, \ldots, \mathbf{G}_{\mathbf{N}}\right\}$.

2. For each matrix $\mathbf{G}_{\mathbf{i}}$, calculate an singular value decomposition $\mathbf{G}_{\mathbf{i}}=\mathbf{U}_{\mathbf{i}} *$ $\boldsymbol{\Sigma}_{\mathbf{i}} * \mathbf{V}_{\mathbf{i}}^{T}$.

3. Generalize soft-thresholding operator with weight vector w:

$$
\operatorname{THRESH}\left(\boldsymbol{\Sigma}_{\mathbf{i}}\right)=\max \left(\boldsymbol{\Sigma}_{i i}-w_{i} / 2,0\right)
$$

where $w_{i}$ is the $i$-th weight of $w$.

4. Update $\mathbf{G}_{\mathbf{i}}$ as $\mathbf{U}_{\mathbf{i}} * \operatorname{THRESH}\left(\boldsymbol{\Sigma}_{\mathbf{i}}\right) * \mathbf{V}_{\mathbf{i}}{ }^{T}$.

5. Update $\hat{\mathbf{X}}$ by putting back updated patches. Overlapped pixels are averaged. (we denote this operation as BACK).

It is easy to see that the above process (also shown in Fig. 3) is an extension of WNNM. The projection will continue iteratively until the stop condition is met. The whole optimization process is summarized in Alg. 1. 


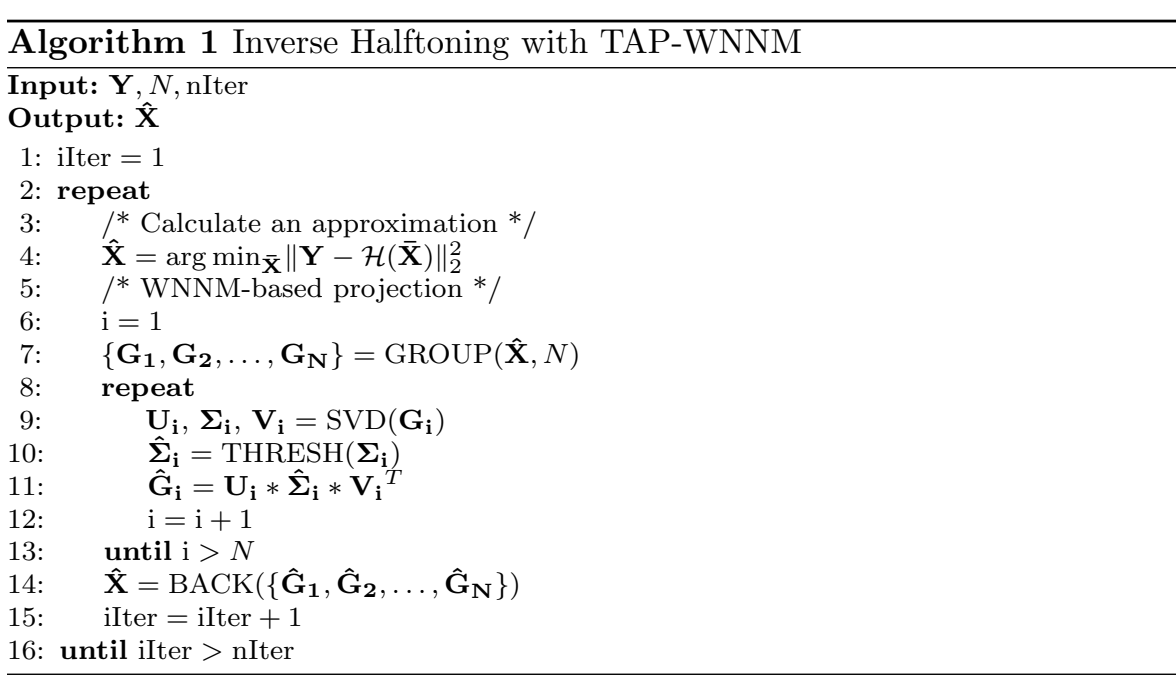

\section{Experiments}

This section starts by investigating the iteration number. Followed by comparing our algorithm with state-of-the-art inverse halftoning algorithms.

\subsection{Data and Implementation}

[Data] Following the standard protocol [16,20,28], several images including Lena, Barbara, Boat, Hill, House, Man and Peppers are used as test images, with their sizes being scaled to $(H \times W=256 \times 256)$. Twelve Kodak images ${ }^{1}$ including first four images Kodim01-04, middle four images Kodim09-12 and last four images Kodim21-24 are converted to gray versions and used, with the sizes being reshaped to $(H \times W=256 \times 256)$. The corresponding halftoned images are obtained by the standard Floyd-Steinberg diffusion [4].

[Implementation] For our framework, the patch size is set to $\frac{H}{32} \times \frac{W}{32}$, i.e., $8 \times 8$. Larger Patch size values would get better performance, but will be slower. $(C=70)$ similar patches centered within $(p \times q=20 \times 20)$ surrounding pixels are found for each of $(N=127 \times 127)$ reference patches. The step between neighbor patches is set to 2 pixels. Though $p, q$ and $N$ can be larger at the expense of running time, we do not observe significant improvements. The optimized initial input is simply set to the smoothed version of the input halftone image.

1 http://r0k.us/graphics/kodak/ 


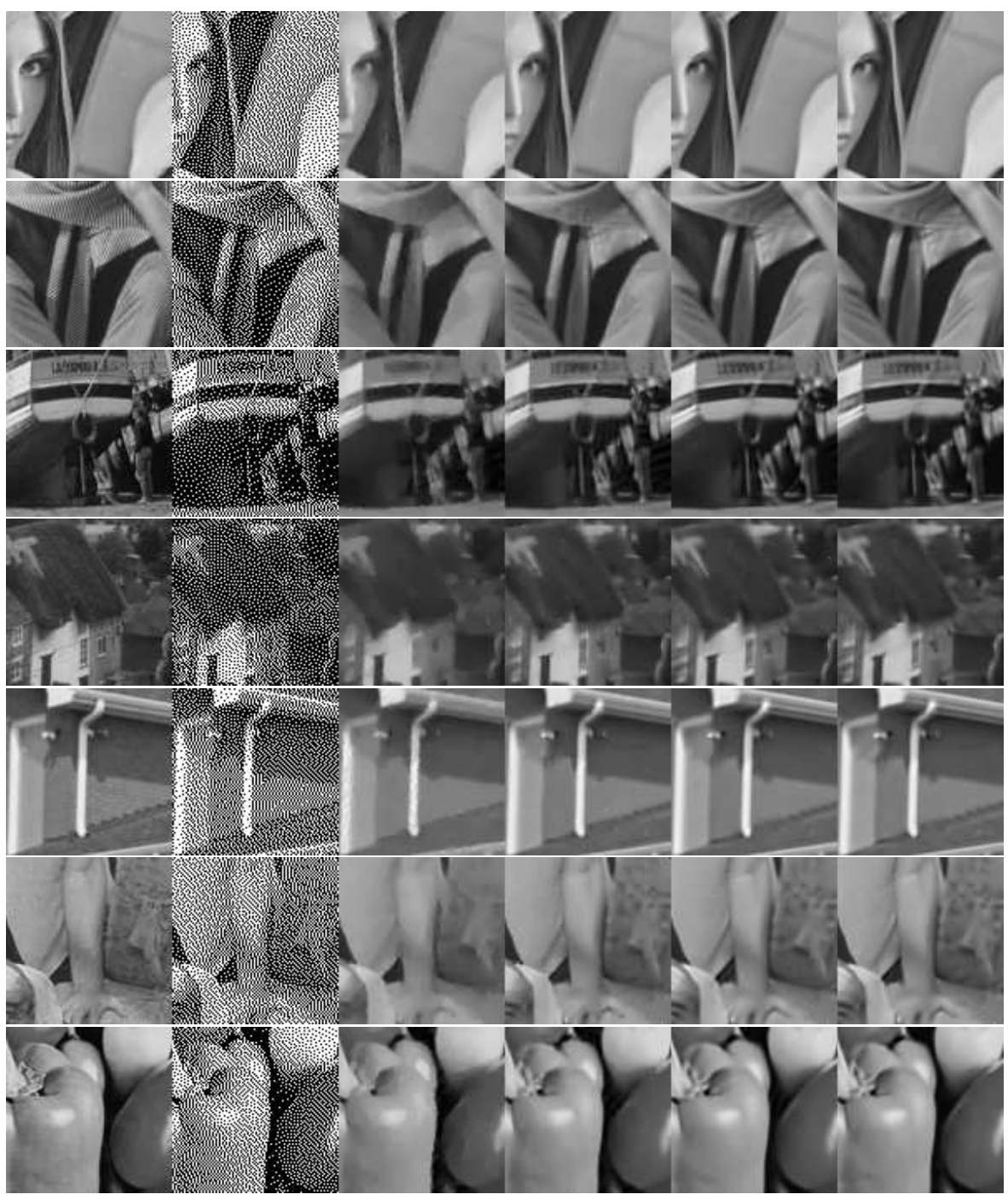

Fig. 4: From-left-to right of the first row: $100 \times 100$ portion of Lena, halftoned, TV-based, BM3D-based [16], G-SVD-based [28] and our results. The second row to the bottom row are for Lena, Barbara, Boat, Hill, House, Man and Peppers respectively.

\subsection{Iteration Number}

We show the effect of the number of iterations. Fig. 6 shows the results of our inverse halftone framework on the first seven test images, with different iterations. We can see that PSNRs and SSIMs increase steadily at first, and then gradually saturate. This phenomenon proves the correctness of the pro- 


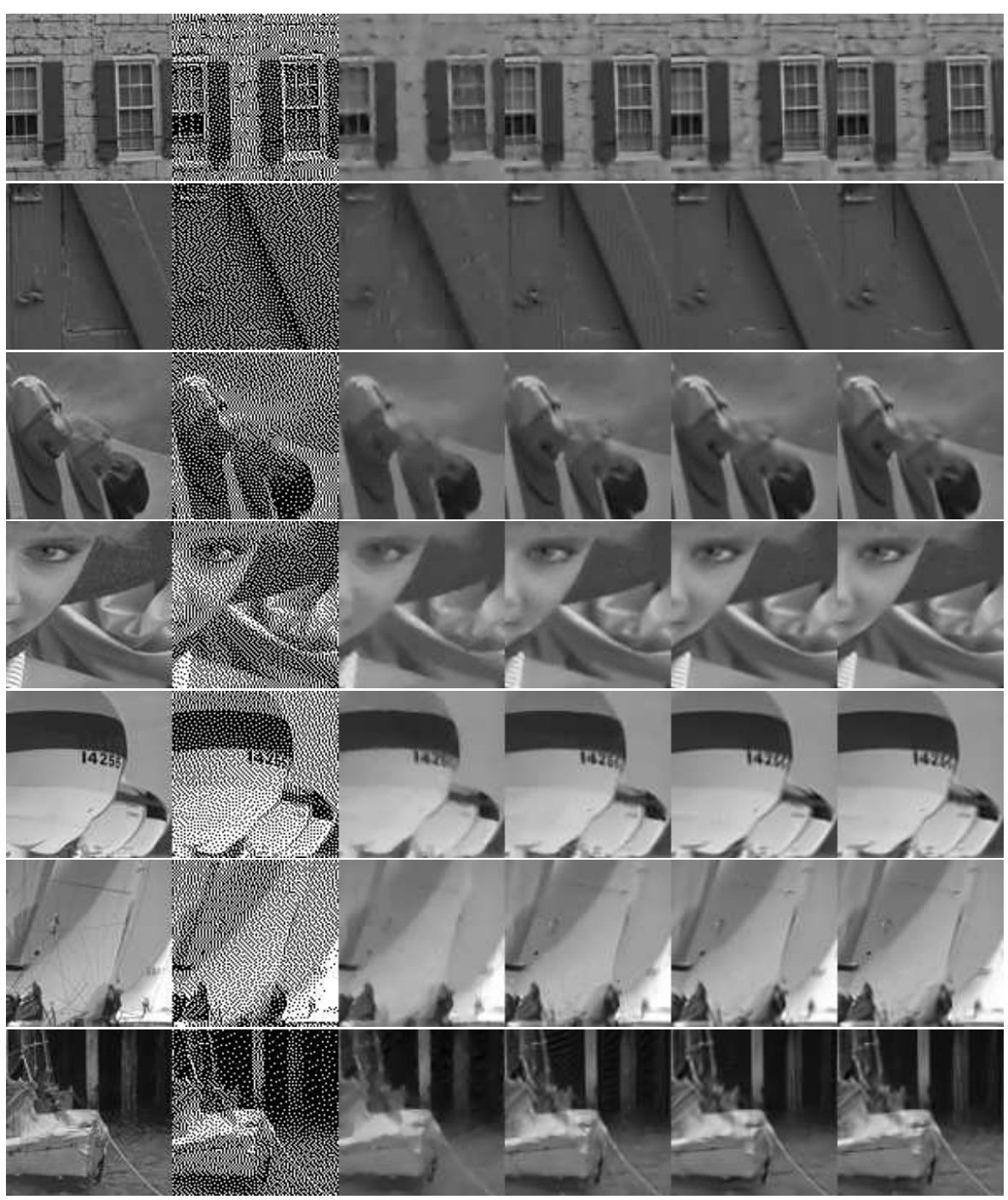

Fig. 5: From-left-to right of the first row: $100 \times 100$ portion of Kodim01, halftoned, TV-based, BM3D-based [16], G-SVD-based [28] and our results. The second row to the bottom row are for Kodim01, Kodim02, Kodim03, Kodim04, Kodim09, Kodim10 and Kodim11 respectively.

posed TAP-WNNM iterative method. As the number of iterations increases, our method gradually improves the quality of the inverse halftoning image, rather than impair the performance. 

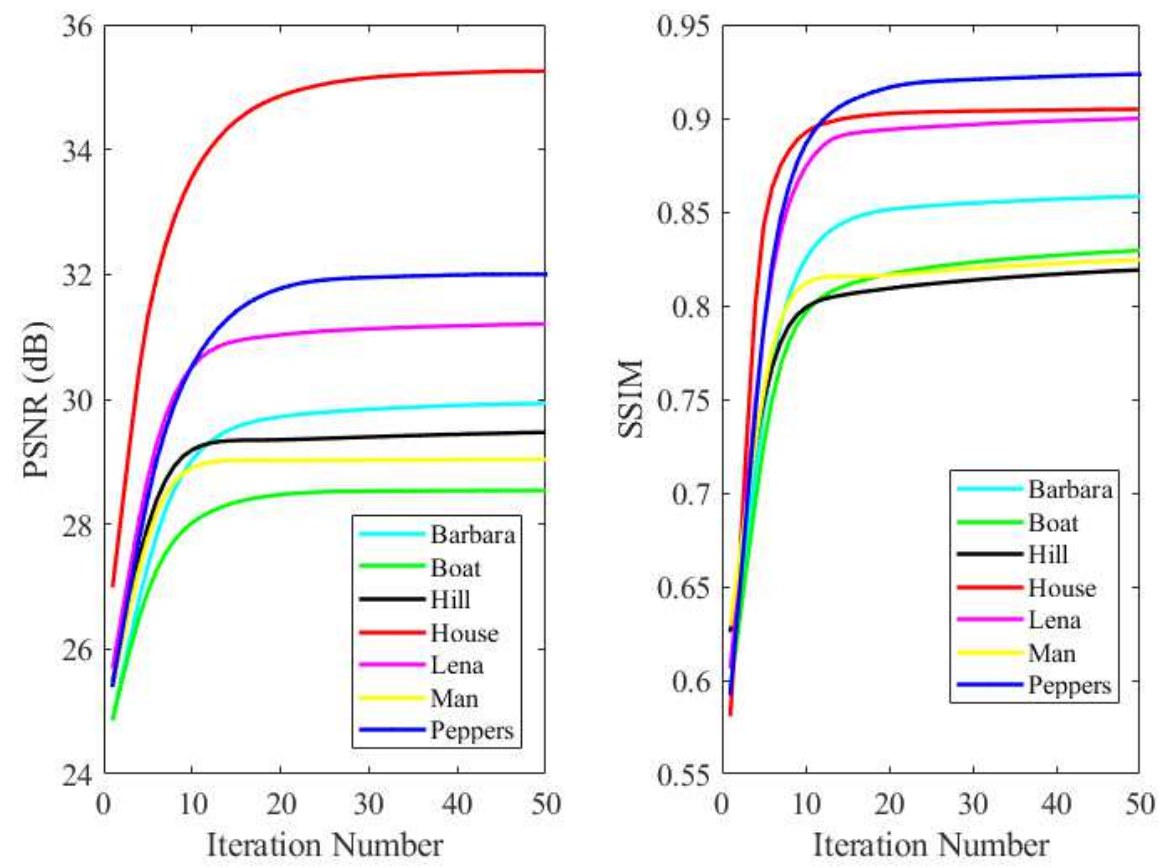

Fig. 6: (a) Test the PSNRs on the images relative to the number of iterations. (b) Test the SSIMs on the images relative to the number of iterations.

\subsection{Comparisons with State of the Arts}

We compare our proposed inverse halftone method with three representative methods: total-variation-based (TV) [15], BM3D based [16] and G-SVD based [28] inverse halftoning.

As shown in Table 1, our framework achieves better or comparable performance when compared with the state-of-arts on all the test images, on the PSNR and SSIM metrics. More precisely, we have obtained the PSNR of more than $29 \mathrm{~dB}$ on Man and kodim11, dramatically outperformed the state-of-thearts. Besides, for the test images House and Man, our framework achieved $35.05 \mathrm{~dB}$ and $29.05 \mathrm{~dB}$ respectively. To the best of our knowledge, this is the first time to see in the open literature that inverse halftoning of House or Man can achieve the PSNR values of larger than $35 \mathrm{~dB}$ and $29 \mathrm{~dB}$ Respectively.

In addition to the Table 1 which contains the objective PSNR / SSIM quality measurement results for all approaches, Fig. 4 and Fig. 5 offer some subjective quality comparisons. Due to the space limitation, we only show results of the first seven test images (e.g. Lena, Barbara, Boat, Hill, House, Man and Peppers in Fig. 4. And the first seven selected Koda test images (e.g. Kodim01, Kodim02, Kodim03, Kodim04, Kodim09, Kodim10 and Kodim11) in 
Table 1: The PSNRs / SSIMs of all frameworks

\begin{tabular}{l|c|c|c|c|}
\hline Image & TV-Based & BM3D based & G-SVD based & Proposed \\
\hline Lena & $28.59 / 0.8478$ & $30.69 / 0.8838$ & $31.09 / 0.8896$ & $\mathbf{3 1 . 1 8 / 0 . 8 9 5 6}$ \\
Barbara & $27.33 / 0.7948$ & $29.41 / 0.8451$ & $29.89 / 0.8549$ & $\mathbf{2 9 . 9 6 / 0 . 8 5 9 3}$ \\
Boat & $26.48 / 0.7454$ & $28.48 / 0.8208$ & $28.41 / 0.8173$ & $\mathbf{2 8 . 5 6 / 0 . 8 3 0 0}$ \\
Hill & $27.73 / 0.7429$ & $29.37 / 0.8161$ & $29.20 / 0.8124$ & $\mathbf{2 9 . 4 8 / 0 . 8 2 0 8}$ \\
House & $31.43 / 0.8773$ & $34.65 / \mathbf{0 . 8 9 6 7}$ & $34.80 / 0.8943$ & $\mathbf{3 5 . 0 5} / 0.8965$ \\
man & $27.41 / 0.7616$ & $28.99 / 0.8187$ & $28.92 / 0.8172$ & $\mathbf{2 9 . 0 5 / 0 . 8 2 5 0}$ \\
peppers & $29.26 / 0.8829$ & $31.65 / 0.9107$ & $31.63 / 0.9093$ & $\mathbf{3 1 . 9 8 / 0 . 9 2 0 1}$ \\
kodim01 & $24.64 / 0.6093$ & $26.89 / 0.7508$ & $26.82 / 0.7482$ & $\mathbf{2 6 . 9 4 / 0 . 7 5 5 0}$ \\
kodim02 & $31.14 / 0.8075$ & $32.87 / 0.8464$ & $32.52 / 0.8409$ & $\mathbf{3 3 . 0 0 / 0 . 8 4 7 8}$ \\
kodim03 & $30.73 / 0.8696$ & $31.92 / 0.8870$ & $31.78 / 0.8830$ & $\mathbf{3 2 . 2 3 / 0 . 8 9 0 7}$ \\
kodim04 & $29.61 / 0.7967$ & $31.31 / 0.8352$ & $31.49 / 0.8310$ & $\mathbf{3 1 . 7 7 / 0 . 8 4 4 0}$ \\
kodim09 & $28.28 / 0.8490$ & $30.41 / 0.8828$ & $30.19 / 0.8874$ & $\mathbf{3 0 . 5 3 / 0 . 8 9 0 0}$ \\
kodim10 & $28.34 / 0.7964$ & $30.15 / 0.8558$ & $30.11 / 0.8547$ & $\mathbf{3 0 . 4 6 / 0 . 8 6 6 8}$ \\
kodim11 & $27.38 / 0.7510$ & $28.93 / 0.7948$ & $28.63 / 0.7987$ & $\mathbf{2 9 . 0 2 / 0 . 8 0 9 8}$ \\
kodim12 & $30.04 / 0.8227$ & $31.64 / 0.8415$ & $31.30 / 0.8344$ & $\mathbf{3 1 . 9 1 / 0 . 8 4 7 6}$ \\
kodim21 & $26.42 / 0.7871$ & $\mathbf{2 7 . 9 7 / 0 . 8 3 0 9}$ & $27.71 / 0.8368$ & $27.81 / \mathbf{0 . 8 4 3 0}$ \\
kodim22 & $28.21 / 0.7113$ & $29.68 / 0.7780$ & $29.53 / 0.7724$ & $\mathbf{2 9 . 8 4 / 0 . 7 8 7 9}$ \\
kodim23 & $29.92 / 0.8716$ & $31.90 / 0.9018$ & $31.78 / 0.8934$ & $\mathbf{3 2 . 1 0 / 0 . 9 0 8 7}$ \\
kodim24 & $25.90 / 0.7134$ & $27.10 / 0.7936$ & $27.02 / 0.7886$ & $\mathbf{2 7 . 2 2 / 0 . 7 9 7 7}$ \\
\hline
\end{tabular}

Fig. 5. We can see that in Barbara, the texture of the scarf is reconstructed with a higher fidelity. And there are lesser artificial flaws in the smooth region of Peppers.

\section{Conclusion}

This paper studies how to build an effective inverse halftone framework. To avoid fuzzy solutions, we propose a TAP-WNM constraint to take advantage of the similarity of nonlocal image patches. Our TAP-WNNM constraint may also be beneficial for related image reconstruct problems, e.g., denoising. Work in these directions are ongoing and will be presented in future.

\section{Compliance with Ethical Standards statements}

Ethical approval: This article does not contain any studies with human participants/animals performed by any of the authors.

Funding details: This work was supported in part by the Zhejiang Public Welfare Technology Research Project Fund of China under Grant LGG20F010010 and GG21F030030, and the City Public Welfare Technology Application Research Project of Jiaxing Science and Technology Bureau of China under Grant 2021AY10071, 2018AY11008, 2020AY10009 and 2021AY10043, and Research Project of Jiaxing Nanhu University under Grant N41472001-YB8.

Conflict of interests: The authors declare that they have no conflict of interest regarding the publication of this article. 
Informed Consent: The authors consent the declarations and the publication of this article.

\section{Authorship contributions}

Jun Yang: Writing original draft, Software, Formal analysis. Xiaojun Jia: Conceptualization, Methodology, Funding acquisition. Zihao Liu: Investigation, Project administration, Data curation. Li Chen and Ying Wu: Supervision, Writing review and editing.

\section{References}

1. Cai, J.F., Candes, E.J., Shen, Z.: A singular value thresholding algorithm for matrix completion. Siam Journal on Optimization 20(4), 1956-1982 (2008)

2. Easley, G.R., Patel, V.M., Healy Jr, D.M.: Inverse halftoning using a shearlet representation. In: SPIE Optical Engineering + Applications, pp. 74460C-74460C. International Society for Optics and Photonics (2009)

3. Eckart, C., Young, G.: The approximation of one matrix by another of lower rank. Psychometrika 1(3), 211-218 (1936)

4. Floyd, R.W.: An adaptive algorithm for spatial gray-scale. In: Proc. Soc. Inf. Disp. vol. 17 , pp. $75-77$ (1976)

5. Gu, S., Lei, Z., Zuo, W., Feng, X.: Weighted nuclear norm minimization with application to image denoising. In: Computer Vision \& Pattern Recognition (2014)

6. Gu, S., Xie, Q., Meng, D., Zuo, W., Feng, X., Zhang, L.: Weighted nuclear norm minimization and its applications to low level vision. International Journal of Computer Vision 121(2), 183-208 (2017)

7. Guo, J., Liu, Y., Chang, J., Lee, J.: Efficient halftoning based on multiple look-up tables (2013)

8. Hein, S., Zakhor, A.: Halftone to continuous-tone conversion of error-diffusion coded images. In: Sigma Delta Modulators, pp. 133-154. Springer (1993)

9. Hou, X., Qiu, G.: Image companding and inverse halftoning using deep convolutional neural networks (2017)

10. Jimenez, F.P., Miyatake, M.N., Medina, K.T., Perez, G.S., Meana, H.P.: An inverse halftoning algorithms based on neural networks and atomic functions. IEEE Latin America Transactions 15(3), 488-495 (2017)

11. Kang, S.J., Do, H.C., Cho, B.G., Chien, S.I., Tae, H.S.: Improvement of low graylevel linearity using perceived luminance of human visual system in pdp-tv. Consumer Electronics, IEEE Transactions on 51(1), 204-209 (2005)

12. Kim, Y.T., Arce, G.R., Grabowski, N.: Inverse halftoning using binary permutation filters. Image Processing, IEEE Transactions on 4(9), 1296-1311 (1995)

13. Kipphan, H.: Handbook of print media: technologies and production methods. Springer (2001)

14. Kite, T.D., Damera-Venkata, N., Evans, B.L., Bovik, A.C.: A fast, high-quality inverse halftoning algorithm for error diffused halftones. Image Processing, IEEE Transactions on 9(9), 1583-1592 (2000)

15. Kite, T.D., Evans, B.L., Bovik, A.C.: Modeling and quality assessment of halftoning by error diffusion. Image Processing, IEEE Transactions on 9(5), 909-922 (2000)

16. Li, X.: Inverse halftoning with nonlocal regularization. In: Image Processing (ICIP), 2011 18th IEEE International Conference on, pp. 1717-1720. IEEE (2011)

17. Liu, Y.F., Guo, J.M., Lee, J.D.: Inverse halftoning based on the bayesian theorem. Image Processing, IEEE Transactions on 20(4), 1077-1084 (2011) 
18. Luo, J., de Queiroz, R., Fan, Z.: A robust technique for image descreening based on the wavelet transform. Signal Processing, IEEE Transactions on 46(4), 1179-1184 (1998)

19. Pelcastre-Jimenez, F., Nakano-Miyatake, M., Toscano-Medina, K., Sanchez-Perez, G. Perez-Meana, H.: An inverse halftoning algorithm based on neural networks and up(x) atomic function. In: International Conference on Telecommunications \& Signal Processing (2015)

20. R. Neelamani, R.N., Baraniuk, R.: Winhd: Wavelet-based inverse halftoning via deconvolution. Rejecta Mathematica 1(6), 84-103 (2009)

21. Rajwade, A., Rangarajan, A., Banerjee, A.: Image denoising using the higher order singular value decomposition. Pattern Analysis and Machine Intelligence, IEEE Transactions on 35(4), 849-862 (2013)

22. Rose, K.: Deterministic annealing for clustering, compression, classification, regression, and related optimization problems. Proceedings of the IEEE 86(11), 2210-2239 (1998)

23. Stevenson, R.L.: Inverse halftoning via map estimation. Image Processing, IEEE Transactions on 6(4), 574-583 (1997)

24. Ting, M.Y., Riskin, E.A.: Error-diffused image compression using a binary-to-gray-scale decoder and predictive pruned tree-structured vector quantization. IEEE transactions on image processing 3(6), 854-858 (1994)

25. Wong, P.W.: Inverse halftoning and kernel estimation for error diffusion. Image Processing, IEEE Transactions on 4(4), 486-498 (1995)

26. Xiong, Z., Orchard, M.T., Ramchandran, K.: Inverse halftoning using wavelets. Image Processing, IEEE Transactions on 8(10), 1479-1483 (1999)

27. Yan, Z., Zhang, E., Chen, W., Chen, Y., Duan, J.: Sparsity-based inverse halftoning via semi-coupled multi-dictionary learning and structural clustering. Engineering Applications of Artificial Intelligence 72, 43-53 (2018)

28. Yang, J., Guo, J., Chao, H.: Inverse halftoning with grouping singular value decomposition. In: IEEE International Conference on Image Processing (2015) 


\section{Figures}

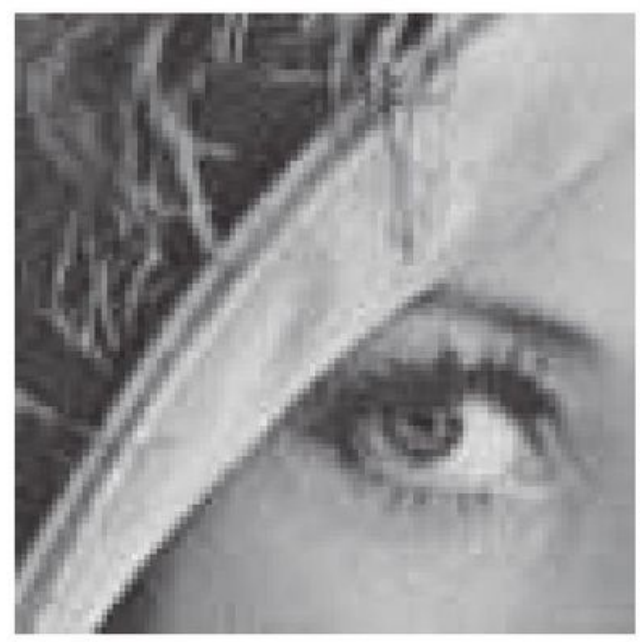

(a)

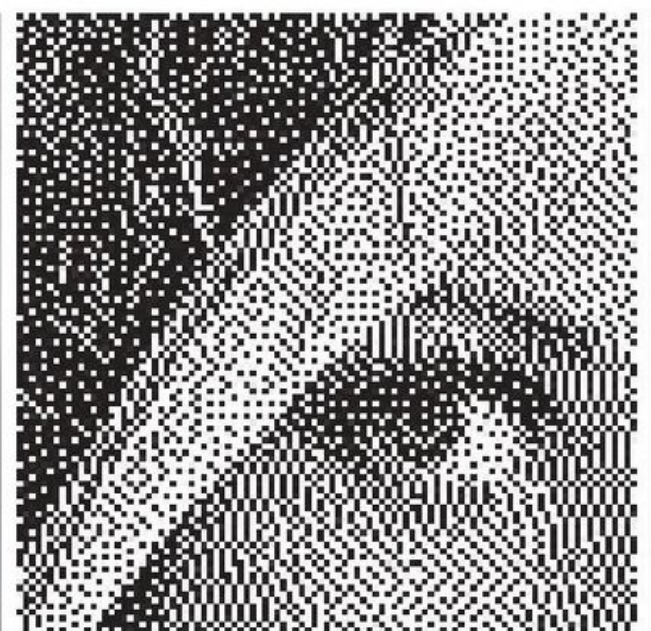

(b)

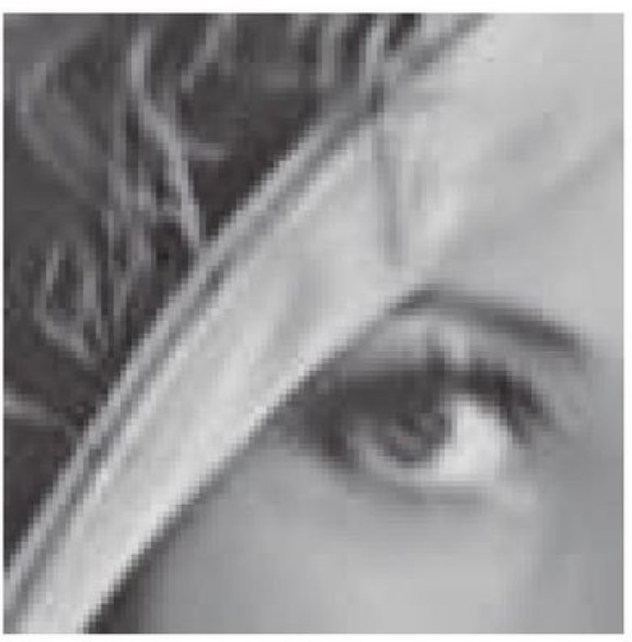

(c)

Figure 1

Please see the Manuscript PDF file for the complete figure caption
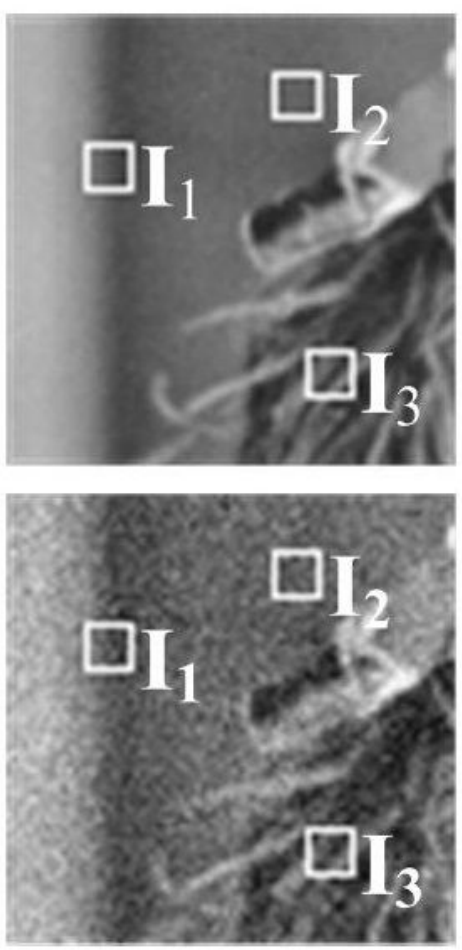

(a)

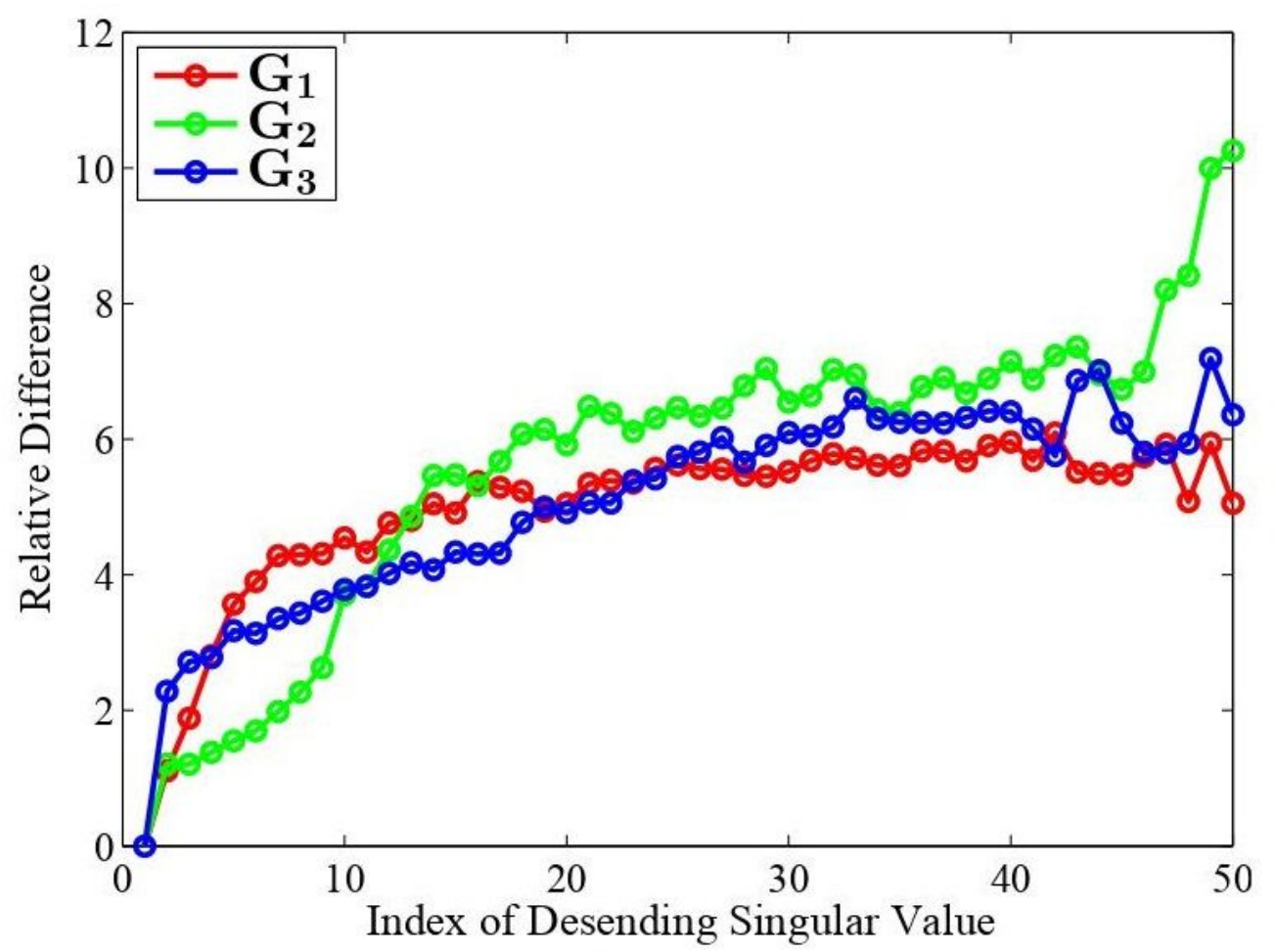

(b)

Figure 2

Please see the Manuscript PDF file for the complete figure caption 


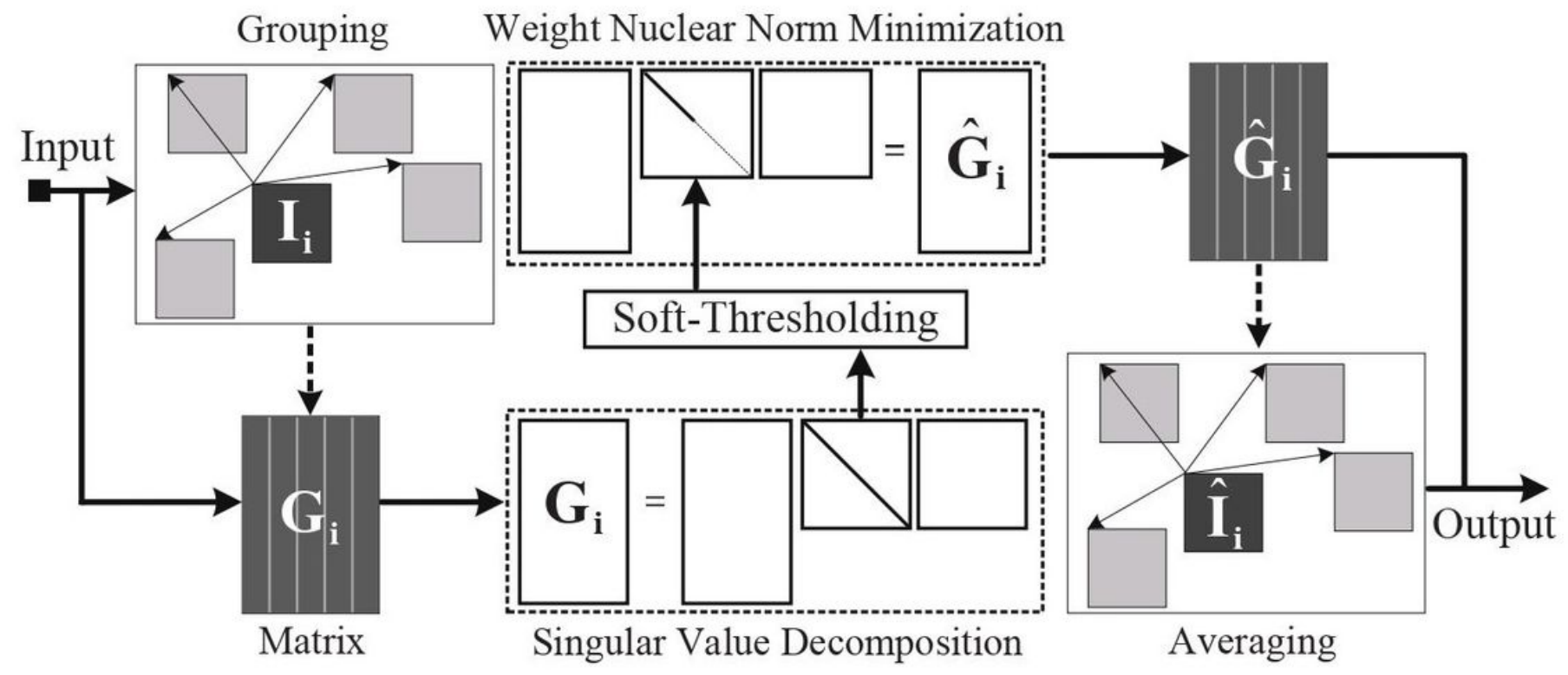

Figure 3

Please see the Manuscript PDF file for the complete figure caption 

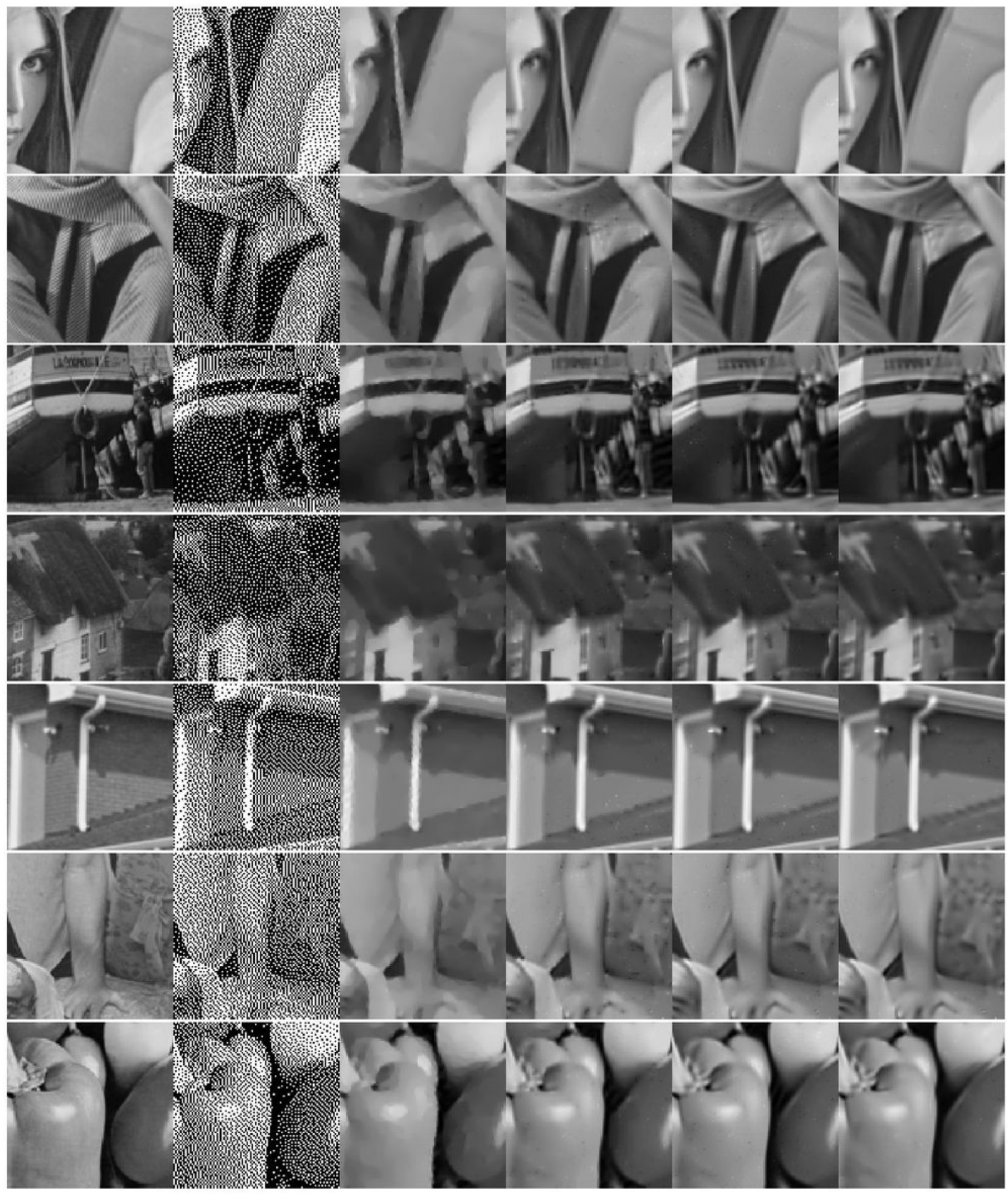

Figure 4

Please see the Manuscript PDF file for the complete figure caption 


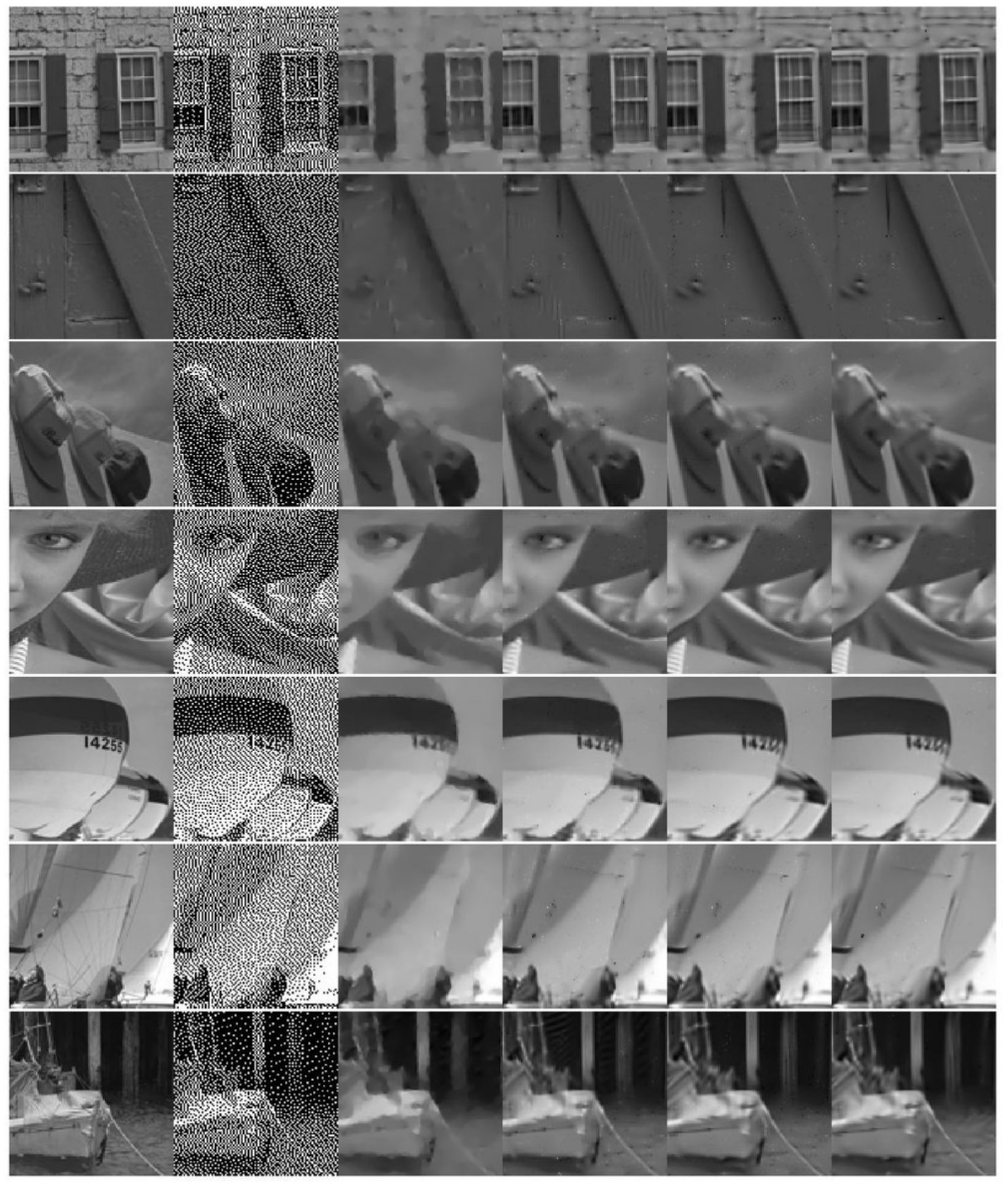

Figure 5

Please see the Manuscript PDF file for the complete figure caption 

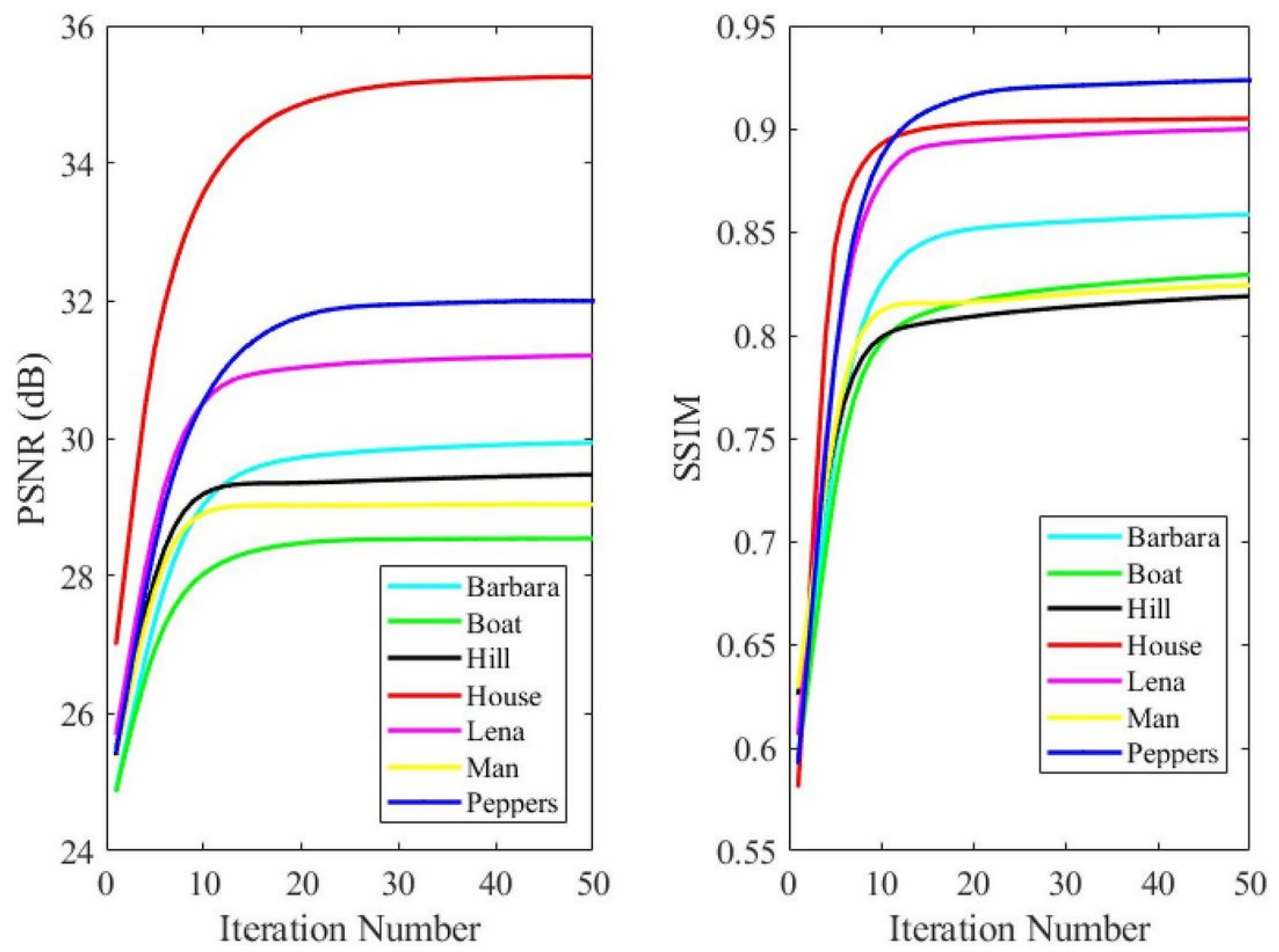

Figure 6

Please see the Manuscript PDF file for the complete figure caption 